\title{
CORRECTION
}

\section{Correction to: Diabetes Technol Ther 2017;19(5):299-304}

In the original article, "Effectiveness of SmartGuard Technology in the Prevention of Nocturnal Hypoglycemia After Prolonged Physical Activity," by Dr. Lenka Petruzelkova, et al., Volume 19, no. 5, 2017, pp 299-304, the correct affiliation of Dr. Klara Pickova has been inadvertently omitted.

On page 299, the correct affiliation has been included as the second footnote:

Klara Pickova, $\mathrm{MD}{ }^{2}$

${ }^{2} 1$ st Department of Internal Medicine, University Hospital and Medical Faculty in Pilsen, Charles University in Prague, Czech Republic.

In the Abstract on the same page, "gS" means "grams of saccharides or carbohydrates".

We have updated the online version to reflect the changes.

We regret the error. 